\title{
Analysis of Vertical and Horizontal Circular C-Arm Trajectories
}

\author{
A. Maier ${ }^{1}$, J.-H. Choi ${ }^{1}$, A. Keil ${ }^{1}$, C. Niebler ${ }^{2}$, M. Sarmiento ${ }^{3}$, A. Fieselmann ${ }^{4}$,

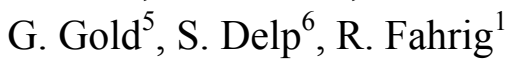 \\ ${ }^{1}$ Radiological Sciences Laboratory (RSL), School of Medicine, Stanford University, USA \\ ${ }^{2}$ Institute of Medical Physics (IMP), Friedrich-Alexander-University \\ Erlangen-Nuremberg, Germany \\ ${ }^{3}$ Siemens AG, Forchheim, Germany \\ ${ }^{4}$ Pattern Recognition Lab and Erlangen Graduate School in Advanced Optical Technologies (SAOT), \\ Department Computer Science, Friedrich-Alexander-University, Erlangen-Nuremberg, Germany \\ ${ }^{5}$ NeuroMuscular Biomechanics Laboratory (NMBL), School of Engineering, \\ Stanford University, USA \\ ${ }^{6}$ Department of Radiology, School of Medicine, Stanford University, USA
}

\begin{abstract}
C-arm angiography systems offer great flexibility in the acquisition of trajectories for computed tomography. Theoretically, these systems are able to scan patients while standing in an upright position. This would allow novel insights into structural changes of the human anatomy while weight bearing. However, this would require a scan on a horizontal trajectory parallel to the ground floor which is currently not supported by standard C-arm CT acquisition protocols.

In this paper, we compared the standard vertical and the new horizontal scanning trajectories by analysis of the source positions and source to detector distances during the scan. We employed a C-arm calibration phantom to compute the exact scan geometry. Based on the analysis of the projection matrices, we computed the source position in 3D and the source to detector distance for each projection. We then used the calibrated scan geometries to reconstruct the calibration phantom. Based on this reconstruction in comparison to the ideal phantom geometry we also evaluated the geometric reconstruction error.
\end{abstract}

As expected, both the vertical and the horizontal scan trajectories exhibit a significant C-arm "wobble". But in both kinds of trajectories, the reproducibility over several scans was comparable. We were able to reconstruct the calibration phantom with satisfactory geometric reconstruction accuracy. With a reconstruction error of $0.2 \mathrm{~mm}$, we conclude that horizontal C-arm scans are possible and show properties similar to those of vertical C-arm scans.

The remaining challenge is compensation for the involuntary movement of the standing subject during a weight-bearing acquisition. We investigated this using an optical tracking system and found that the average movement at the knee while standing upright for 5 seconds is between $0.42 \mathrm{~mm}$ and $0.54 \mathrm{~mm}$, and goes up to as much as $12 \mathrm{~mm}$ when the subject is holding a $60^{\circ}$ squat. This involuntary motion is much larger than the reconstruction accuracy. Hence, we expect artifacts in reconstructions to be significant for upright positions, and overwhelming in squat positions if no motion correction is applied.

Medical Imaging 2011: Physics of Medical Imaging, edited by Norbert J. Pelc, Ehsan Samei, Robert M. Nishikawa, Proc. of SPIE Vol. 7961, 796123 - (C) 2011 SPIE · CCC code: 1605-7422/11/\$18 - doi: 10.1117/12.878502 


\section{INTRODUCTION}

Accurate measurement of relative bone location (i.e. kinematics) in vivo during weight-bearing activities provides valuable insight into the functioning of healthy and injured joints. The knee joint is particularly susceptible to injury as it is subjected to high loads and complex interactions between the associated structures. The etiology of several knee disorders, such as patellofemoral (PF) pain, remain unclear; this is reflected in the different clinical assessments and surgical interventions [1][2][3][4] used in treating PF pain, which vary in efficacy in alleviating symptoms and improving quality of life [5]. Recent developments in C-Arm CT technology have enabled low- and high-contrast imaging and 3D reconstruction of anatomic structures [6][7][8][9], providing a promising technique to characterize the knee joint under loaded conditions.

With access to hardware sub-system controls on a C-Arm system, we were able to acquire data in a horizontal scan plane (i.e. with the axis of rotation perpendicular to the floor) for the first time as shown in

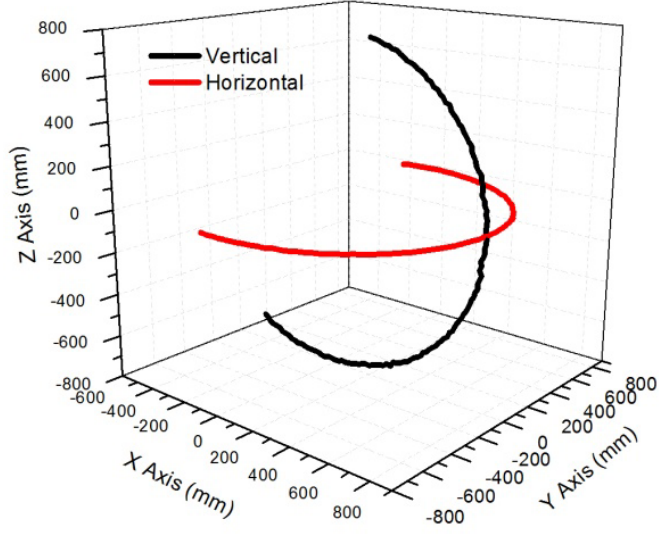

Figure 1. X-ray source positions in 3D space for vertical and horizontal scans.

Fig. 1. Fig. 2 shows sagittal view images of 3-D reconstruction of a cadaver leg acquired with a horizontal scan trajectory. In theory, reconstruction of a horizontal and a vertical scan are exactly the same. In practice, however, a horizontal scan may introduce additional challenges to the reconstruction as external forces such as gravity may have substantial influence on the properties of the C-arm system. Hence, it is not guaranteed that a horizontal scan will be of the same quality as the well-known vertical trajectories.

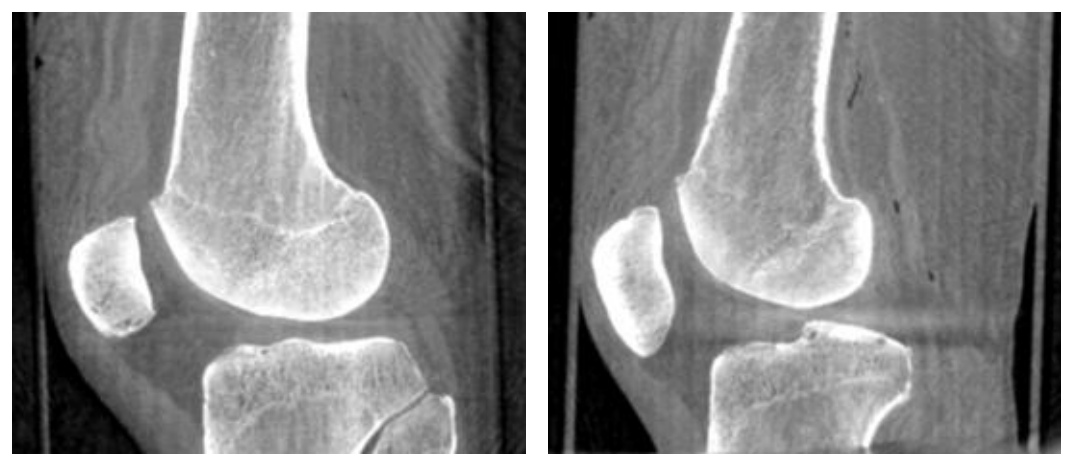

Figure 2. 3-D reconstruction of a cadaver leg (left: no pressure, right: pressure applied) with scan protocol of 5 second scan time, $1.2 \mu \mathrm{Gy}$ radiation dose, 133 projections, $70 \mathrm{kVp}$ tube voltage, and $960 \times 1024$ resolution.

\section{MATERIALS AND METHODS}

\section{Calibration}

Calibration of the projection matrices is performed by linking the known coordinates of a set of 3-D points and their cone-beam projections. In order to solve the correspondence problem between 2-D and 3-D points, we used the PDS-2 calibration phantom [10]. The PDS-2 phantom has 108 beads of two different radii embedded in the shape of a helix on the outer surface of a hollow acrylic cylinder. The cylinder itself is relatively transparent in the X-ray projections. The alternating order of large and small beads is defined in such a manner that any position along the helix can be determined from a sub-sequence of eight consecutive beads. 
The PDS-2 phantom was carefully positioned so that the projected bead spiral does not self-intersect in the projection data. In a first step, the beads were extracted from the cone-beam projections in order to determine their positions with respect to the detector. Second, all beads in the cone-beam projections are mapped to their 3D counterparts by decoding binary sub-sequences of eight beads. As a last step, a projection matrix is estimated for each given source-detector position using the mapping information from the previous step. The calibration algorithm is described in more detail in [10].

\section{Source Position Analysis}

A geometric analysis of the $3 \times 4$ projection matrix $P$ yields the condition $P \cdot \overrightarrow{\mathrm{s}_{\mathrm{h}}}=0$ on the location of the source position $\overrightarrow{\mathrm{s}}=(\mathrm{x}, \mathrm{y}, \mathrm{z})$ represented as a 4-vector of homogeneous coordinates $\overrightarrow{\mathrm{s}}=(\mathrm{x}, \mathrm{y}, \mathrm{z}, 1)$, see [11]. Partitioning the projection matrix into $P=\left(M \mid \vec{p}_{4}\right)$, where $M$ is a $3 \times 3$ matrix and $\vec{p}_{4}$ is a 3-vector, this condition results in the equation

$$
\overrightarrow{\mathrm{s}}=-\mathrm{M}^{-1} \cdot \overrightarrow{\mathrm{p}}_{4}
$$

for the source position $\vec{s}$. Each scan trajectory was repeated several times with movements of the C-arm in between to determine the reliability of the source trajectory.

The calibration phantom's coordinate system cannot be used as a reference frame since the phantom and/or the C-arm are moved in between the scans. Therefore, an intrinsic reference frame has to be defined for evaluating the C-arm trajectories. This is achieved by fitting a plane to all source positions (minimizing their squared distances to that plane) using a principal component analysis [12] [13].

\section{Source to Detector Distance}

The matrix $\mathrm{K}$ is a $3 \times 3$ intrinsic camera matrix with positive focal lengths $f_{x}$ and $f_{y}$ in pixels. (These should be equal for perfectly square pixels.) The focal length is the distance from the camera center to the principal point (?????) on the image plane. The source to detector distance (in $\mathrm{mm}$ ) in world coordinate dimensions can be computed conveniently as the product of $f_{x}$ and a given pixel size in camera u-direction or the product of $f_{y}$ and a given pixel size in camera vdirection. The results of the two methods should, for a well-calibrated matrix K, be equal.

\section{Reconstruction Error Analysis}

The performance of the reconstruction algorithm was evaluated by assessing the reconstructed image error. We defined a reconstruction error as the deviation in the coordinates between the reconstructed calibration bead positions and their positions as defined in the phantom specification of two disjoint calibration runs. In order to determine the bead positions in the reconstruction, we chose a threshold to get points for bead candidates. The actual bead positions were then computed as the geometric center of the point candidate positions. The reconstruction error was then calculated as:

$$
\text { Restruction error }(\mathrm{mm})=\frac{1}{\mathrm{~N}} \cdot \sum_{1}^{\mathrm{N}}\left\|\overrightarrow{\mathrm{C}}_{\text {phantom }}-\overrightarrow{\mathrm{C}}_{\text {reconstructed }}\right\| \text {, }
$$

where $\vec{C}$ denotes $3 \mathrm{D}$ coordinates, $\|\cdot\|$ the Euclidean distance, and $\mathrm{N}$ is the total number of the PDS-2 phantom beads. In addition, the Krippendorff-Lin concordance correlation coefficient [12][13] was also measured to provide the agreement between the coordinates of beads in the phantom and the reconstructed image. 


\section{Experimental Setup}

The vertical and horizontal scans of the PDS-2 phantom were carried out on a C-arm CT scanner (Axiom Artis dTA, Siemens AG, Forchheim, Germany) using a digital flat panel detector $(30 \mathrm{~cm} \times 40 \mathrm{~cm}, 0.158 \mathrm{~mm} \times 0.158 \mathrm{~mm}$ pixels native).

Images were acquired while the $\mathrm{C}$-arm rotated around the PDS- 2 phantom at a rate of $1.5^{\circ}$ per projection, with $2 \times 2$ pixel binning, resulting in 133 images. The tube voltage was $70 \mathrm{kVp}$, the total scan time was 5 seconds, and the radiation dose was $1.2 \mu$ Gy per projection. The PDS-2 phantom was scanned vertically and horizontally. The phantom was positioned near the center of the scan. For the case of the vertical scan the main axis of the helix was aligned approximately parallel to the floor, in the case of the horizontal scan the main axis was approximately perpendicular to the floor (see Fig 1).

In order to quantify the expected involuntary motion of a real patient during a scan, we used an optical tracking system (Vicon Motion Systems, Oxford, UK) to track healthy volunteers while standing and also with knees somewhat bent (i.e. in semi-squat positions). The duration of the sequences was 5 seconds which is the length of a typical single sweep scan. The data were sampled at $120 \mathrm{~Hz}$ and markers were attached to the left and right knee.

\section{RESULTS}

\section{C-Arm Wobble / Trajectory Characteristics}

The analysis of the source position deviations and the source to detector distances are shown in Table 1 and Figures 3 and 4 , comparing the two different scan modes.

Table 1. Analysis of the source position deviation and the source to detector distance.

\begin{tabular}{|l|c|c|c|c|}
\hline Scan mode & Source position to C-arm rotation & \multicolumn{3}{|c|}{ Source to detector distance [mm] } \\
\cline { 3 - 5 } & plane distance $[\mathrm{mm}]$ & Mean & $|\max -\min |$ & $\sigma_{\mathrm{SD}}$ \\
\hline Vertical & 0.2946 & 1193.88 & 45.16 & 9.75 \\
\hline Horizontal & 0.2966 & 1199.98 & 28.87 & 5.70 \\
\hline
\end{tabular}

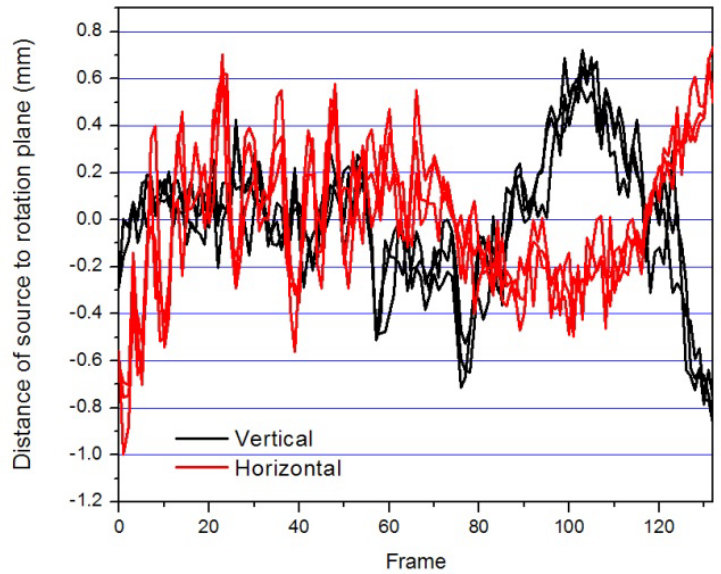

Figure 3. Distances of source positions to the C-arm rotation plane along frame indices for 3 vertical and 3 horizontal scans.

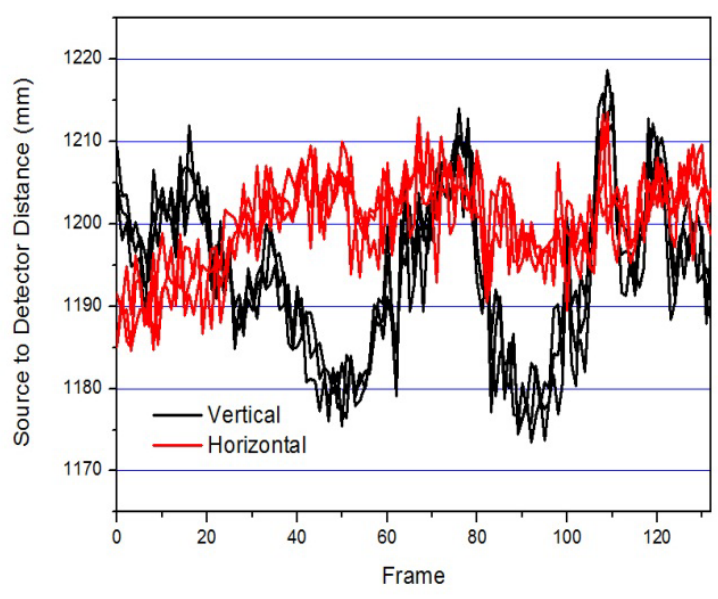

Figure 4. Source to detector distance along frame indices for 3 vertical and 3 horizontal scans. 
We found that the X-ray source positions deviate up to $1 \mathrm{~mm}$ from the source plane in both vertical and horizontal mode. The variation of the source to detector distance is smaller in the horizontal scan than in the vertical scan. A possible explanation for this is that gravity forces on the $\mathrm{C}$-arm system have a bigger bending effect on the $\mathrm{C}$-arm in the vertical scan. This first analysis shows that horizontal scan planes have similar characteristics as the established vertical ones. What really matters for an exact reconstruction, however, is not how much a C-arm "wobbles" during acquisition but how repeatable its trajectory is. This determines the reconstruction accuracy when using a prior calibration run for determining the acquisition geometry used during subsequent reconstructions.

\section{Trajectory Repeatability / Calibration Validity}

Table 2.1. Analysis of the repeatability of the calibration: We calibrated the horizontal scan on three different days within one week. The results indicate that the trajectory lies in the same range in all calibration scans.

\begin{tabular}{|c|c|c|c|c|}
\hline \multirow{2}{*}{ Scan sets } & \multicolumn{2}{|c|}{$\begin{array}{c}\text { Source position to } \\
\text { C-arm rotation plane distance } \\
\text { variation at each frame [mm] }\end{array}$} & \multicolumn{2}{c|}{$\begin{array}{c}\text { Source to detector distance } \\
\text { variation at each frame [mm] }\end{array}$} \\
\cline { 2 - 5 } & $\sigma_{\mathrm{SD}}$ & $\max -\min$ & $\sigma_{\mathrm{SD}}$ & $\max -\min \mid$ \\
\hline Day 1 (3 sets) & 0.0855 & 0.2918 & 2.4267 & 28.87 \\
\hline Day 2 (3 sets) & 0.0797 & 0.3001 & 2.3546 & 31.55 \\
\hline Day 7 (3 sets) & 0.0771 & 0.2336 & 2.2001 & 32.44 \\
\hline All sets (9 sets) & 0.1170 & 0.1990 & 4.5130 & 34.09 \\
\hline
\end{tabular}

Table 2.2. Analysis of the repeatability of the calibration: The first and last scan sets in a day or a week are used. The results indicate that the trajectory lies in the same range in all calibration scans.

\begin{tabular}{|l|c|c|c|c|}
\hline \multicolumn{1}{|c|}{ Scan sets } & \multicolumn{2}{c|}{$\begin{array}{c}\text { Absolute repositioning errors } \\
\text { perpendicular to the rotation plane } \\
{[\mathrm{mm}]}\end{array}$} & $\begin{array}{c}\text { Absolute error in source to detector } \\
\text { distance [mm] }\end{array}$ \\
\cline { 2 - 5 } & mean & $\max$ & mean & $\max$ \\
\hline Vertical, Intraday & 0.1152 & 0.3914 & 3.4772 & 13.2815 \\
\hline Horizontal, Intraday & 0.1374 & 0.7310 & 3.6579 & 14.6042 \\
\hline Horizontal, Week & 0.1727 & 0.5854 & 6.7891 & 22.2379 \\
\hline
\end{tabular}

Table 2 shows the results of multiple calibration scans on three different days within one week. The source position to Carm rotation plane distance variation and source to detector distance variation at each frame in Table 2.1 are defined as a standard deviation from the mean of the distance at each source position. Then, the variations for all source positions are averaged to obtain the table entries. The source position to C-arm rotation plane distance difference and source to detector distance difference at each frame in Table 2.2 are computed as a difference between two scans on the same day or at the beginning and end of a whole week, respectively. The intraday repeatability of vertical and horizontal acquisition trajectories is comparable. The horizontal acquisition mode has a higher variability in the source to detector distance when evaluated over a whole week. This measure, however, is not as crucial as the source position variation perpendicular to the rotation plane for reconstruction. 


\section{Reconstruction Error}

Table 3. The analysis of reconstruction error

\begin{tabular}{|l|c|c|}
\hline Scan mode & $\begin{array}{c}\text { Reconstruction error } \\
{[\mathrm{mm}]}\end{array}$ & $\begin{array}{c}\text { Concordance } \\
\text { Correlation }\end{array}$ \\
\hline Vertical & 0.37 & 0.99999892 \\
\hline Horizontal & 0.20 & 0.99999687 \\
\hline
\end{tabular}

The assessment of the reconstructed image error is shown in Table 3. In both scan modes, the concordance correlation coefficient is very close to +1 . Thus, the reconstructed bead positions and the known bead positions in the phantom have a very strong positive linear correlation. In both modes, the reconstruction error is in the same range as the detector resolution in $2 \times 2$ binning mode $(0.316 \mathrm{~mm}$ pixel size $)$.

\section{Patient Motion}

Table 4 shows the results of the movement at the right and left knee of healthy volunteers with and without knee flexion.

Table 4. Results of the movement at the right and left knee: We used an optical tracking system to track the knee movement of 9 healthy volunteers while standing in a bent/squat position. For comparison purposes, the results of one subject with no keen flexion is shown in the bottom row.

\begin{tabular}{|l|c|c|c|c|}
\hline \multirow{2}{*}{} & \multicolumn{2}{|c|}{ Right knee deviation $[\mathrm{mm}]$} & \multicolumn{2}{c|}{ Left knee deviation [mm] } \\
\cline { 2 - 4 } & mean & $\max$ & mean & $\max$ \\
\hline Subject 1 & 2.2440 & 7.2133 & 2.2711 & 6.4410 \\
\hline Subject 2 & 3.4742 & 12.3328 & 3.9625 & 12.9543 \\
\hline Subject 3 & 3.6002 & 6.5330 & 1.5767 & 5.0270 \\
\hline Subject 4 & 1.9458 & 4.8194 & 2.6529 & 6.5446 \\
\hline Subject 5 & 1.7638 & 5.5218 & 2.2409 & 5.5917 \\
\hline Subject 6 & 1.5226 & 6.7000 & 2.1780 & 6.7165 \\
\hline Subject 7 & 1.7899 & 3.6448 & 1.9114 & 4.5860 \\
\hline Subject 8 & 2.4849 & 7.6244 & 2.8671 & 6.4879 \\
\hline Subject 9 & 2.1124 & 5.6450 & 2.5368 & 6.6407 \\
\hline All subjects & 2.3265 & 12.3328 & 2.4664 & 12.9543 \\
\hline Subject 1 with no flexion & 0.4191 & 0.8567 & 0.5436 & 1.4650 \\
\hline
\end{tabular}

A subject with no flexion showed an average motion of $0.42 \mathrm{~mm}$ at the right knee and $0.54 \mathrm{~mm}$ at the left knee. In order to understand knee kinematics in weight-baring positions, we tracked 9 subjects holding a squat at 60 degree of knee flexion for 20 seconds. Nine Subjects with flexion showed an average motion of $2.33 \mathrm{~mm}$ at the right knee and $2.47 \mathrm{~mm}$ at the left knee which is about six to ten times larger than the reconstruction error computed in the last section. Hence, we expect considerable motion artifact which will be even worse in patients with knee pain.

\section{CONCLUSIONS}

In this study, the impact of non-ideal geometrical alignment in vertical and horizontal scan modes has been studied. The source position deviation and the source to detector distance are considered important parameters to describe the scan geometry. Results show that the $\mathrm{C}$-arm gantry shows a variation of the source position of about one millimeter during the 
scan. In both scan modes the reproducibility of the trajectories was comparable. Based on the assessment of the reconstructed image error, the reconstruction image accuracy was also similar in both scan modes. Hence, C-arm CT reconstruction is also possible on horizontal trajectories. In summary, horizontal C-arm scans offer the opportunity to scan subjects in standing, i.e., weight-bearing, position. The image data acquired with the horizontal scan trajectory was of very good quality and will be used to generate weight-bearing volumetric images for the clinical assessment of joint stresses in the future. For the clinical application, however, additional motion compensation will be required, as the average movement of the knee is considerably higher than the reconstruction error. In the clinical setup this will be even more problematic, as we expect more motion from patients with actual knee pain.

\section{ACKNOWLEDGEMENTS}

This work was supported by National Institutes of Health (NIH grant 1R01HL087917), by Siemens AG, Healthcare Sector, and by the Lucas Foundation. This work was never submitted, published, or presented before. The concepts and information presented in this paper are based on research and are not commercially available.

\section{REFERENCES}

[1] E. Servien, P.C. Verdonk, and P. Neyret, "Tibial tuberosity transfer for episodic patellar dislocation," Sports Med Arthrosc, vol. 15, no. 2, pp. 61-7, 2007.

[2] G. L. Camanho, C. Ade Viegas, M.K. Demange, and A. J. Hernandez, "Conservative versus surgical treatment for repair of the medial patellofemoral ligament in acute dislocations of the patella," Arthroscopy, vol. 25, no. 6, pp. $620-5,2009$.

[3] S. T. Donell, G. Joseph, C. B. Hing and T. J. Marshall, "Modified Dejour trochleoplasty for severe dysplasia: operative technique and early clinical results," Knee, vol. 13, no. 4, pp. 266-73, 2006.

[4] J. McConnell, "The physical therapist's approach to patellofemoral disorders," Clin Sports Med, vol. 21, no. 3, pp. 363-87, 2002.

[5] G. Nimon, D. Murray, M. Sandow, and J. Goodfellow, "atural history of anterior knee pain: a 14- to 20-year followup of nonoperative management," J Pediatr Orthop, vol. 18, no. 1, pp. 118-22, 1998.

[6] R. Fahrig, A. J. Fox, S. Lownie, and D. W. Holdsworth, "Use of a C-arm system to generate true three-dimensional computed rotational angiograms: preliminary in vitro and in vivo results," AJNR Am J Neuroradiol, vol. 18, pp. 1507-14, 1997.

[7] R. Fahrig, R. Dixon, T. Payne, R. L. Morin, A. Ganguly, and N. Strobel, "Dose and image quality for a cone-beam C-arm CT system," Med Phys, vol. 33, pp. 4541-50, 2006.

[8] F. Geiger, and D. Parsch, "Intraoperative assessment of femoral component rotational alignment in total knee arthroplasty," Arch Orthop Trauma Surg, vol. 128, no. 3, pp. 267-70, 2008.

[9] U. Linsenmaier, C. Rock, E. Euler, S. Wirth, R. Brandl, D. Kotsianos, W. Mutschler, and K. Pfeifer, "Threedimensional CT with a modified C-arm image intensifier: feasibility," Radiology, vol. 224, no. 1, pp. 286-92, 2002.

[10] S. Hoppe, Accurate Cone-Beam Image Reconstruction in C-Arm Computed Tomography, PhD Thesis ed. Erlangen, Germany: University Erlangen Nuremberg, 2008.

[11] R. Hartley and A. Zisserman, Multiple View Geometry in computer vision, 2nd ed. Cambridge, United Kingdom: Cambridge Univ. Press, 2003.

[12] K. Pearson, "On lines and planes of closest fit to systems of points in space," Philosophical Mag., vol. 2, no. 6, pp. 
559-572, 1901.

[13] H. Hotelling, "Analysis of a complex of statistical variables into principal components," J. of Educational Psychology, no. 24, pp. 417-441, 1933.

[14] K. Krippendorff, "Bivariate agreement coefficients for reliability of data," Sociological Methodology, vol. 2, pp. 139-50, 1970.

[15] L. I.-K. Lin, "A concordance correlation coefficient to evaluate reproducibility," Biometrics, vol. 45, no. 1, pp. $255-$ 68, 1989.

[16] T. F. Besier, G. E. Gold, G. S. Beaupre, and S. L. Delp, "A modeling framework to estimate patellofemoral joint cartilage stress in vivo," Med Sci Sports Exerc, vol. 37, no. 11, pp. 1924-30, 2005. 